\title{
Neuroprotective effects of quercetin in a mouse model of brain ischemic/reperfusion injury via anti-apoptotic mechanisms based on the Akt pathway
}

\author{
XIAOMING LEI $^{1}$, HAILIAN CHAO ${ }^{1}$, ZHENNI ZHANG $^{1}$, JIANRUI LV ${ }^{1}$, SIYUAN LI ${ }^{1}$, \\ HAIDONG WEI ${ }^{1}$, RONGLIANG XUE ${ }^{1}$, FANG LI ${ }^{1}$ and ZONGFANG $\mathrm{LI}^{2}$ \\ Departments of ${ }^{1}$ Anesthesiology and ${ }^{2}$ General Surgery, Second Affiliated Hospital, \\ Xi'an Jiaotong University, Xi'an, Shaanxi 710004, P.R. China
}

Received July 25, 2014; Accepted April 20, 2015

DOI: $10.3892 / \mathrm{mmr} .2015 .3857$

\begin{abstract}
The present study provided experimental evidence for the neuroprotective effects of quercetin using a rat model of global brain ischemic/reperfusion (I/R) injury. Pre-treatment with quercetin (5 or $10 \mathrm{mg} / \mathrm{kg}$ orally (p.o.); once daily) induced a dose-dependent reduction in I/R-induced hippocampal neuron cell loss, with $10 \mathrm{mg} / \mathrm{kg} /$ day being the lowest dose that achieved maximal neuroprotection. Administration of $10 \mathrm{mg} / \mathrm{kg}$ quercetin over at least 3 days prior to $\mathrm{I} / \mathrm{R}$ was required to improve the survival rate of I/R rats. Fluorescence-assisted cell sorting, hematoxylin and eosin staining and terminal deoxynucleotidyl transferase dUTP nick end labeling indicated neuronal cell loss in the CA1 hippocampus. Rats that had undergone transient global cerebral ischemia for 15 min followed by $1 \mathrm{~h}$ of reperfusion exhibited a significant increase in reactive oxygen species (ROS) production in the hippocampus. The I/R-induced ROS overproduction in the hippocampus at 1, 12 and $24 \mathrm{~h}$ following I/R was significantly decreased by quercetin pre-treatment. Western blot analysis revealed that the neuroprotective effects of quercetin (5 and $10 \mathrm{mg} / \mathrm{kg} /$ day, p.o.) were associated with an upregulation of the I/R-induced suppression of B-cell lymphoma-2 (Bcl-2), Bcl extra large and survivin expression as well as phosphorylation of Bcl-2-associated death promoter. Furthermore, the neuroprotective effects of quercetin $(5,10 \mathrm{mg} / \mathrm{kg} / \mathrm{day})$ in the brain were associated with an upregulation of Akt signaling. These
\end{abstract}

Correspondence to: Professor Zongfang Li, Department of General Surgery, Second Affiliated Hospital, Xi'an Jiaotong University, 157 West Fifth Road, Xi'an, Shaanxi 710004, P.R. China E-mail: 1zf2568@gmail.com

Dr Fang Li, Department of Anesthesiology, Second Affiliated Hospital, Xi'an Jiaotong University, Xi'an, Shaanxi 710004, P.R. China

E-mail: xjtulifang@163.com

Key words: reactive oxygen species, quercetin, neuroprotection, global cerebral ischemia/reperfusion, Akt findings suggested that the inhibition of I/R-induced brain injury by quercetin likely involves a transcriptional mechanism to enhance anti-apoptotic signaling.

\section{Introduction}

Flavonoids from natural biological sources, including tea and fruit, and have attracted considerable attention for their applicability in the prevention and treatment of stroke (1). Epidemiological evidence supported the therapeutic potential of these compounds in a variety of pre-clinical models of ischemic brain injury (2). Quercetin is a flavonoid and an important polyphenolic antioxidant (Fig. 1) (3). It was reported that quercetin was able to maintain blood-brain barrier integrity by scavenging active oxygen (4). It was suggested that oral treatment with quercetin nanocapsules may exert a protective effect against oxidative damage and prevent pyramidal neurons in the hippocampus from ischemia/reperfusion (I//R) (5). However, the exact mechanism of the neuroprotective effect of quercetin against global brain I/R injury has remained elusive.

Apoptosis is one of the processes leading to cell death after cerebral ischemia (6). In response to mitochondrial oxidative stress, the mitochondrial outer membrane becomes permeabilized (7), which leads to the translocation of B-cell lymphoma 2 (Bcl-2)-associated $\mathrm{X}$ protein ( $\mathrm{Bax}$ ) from the cytoplasm to the mitochondria, eventually causing the release of cytochrome $c$ (8). Oxidative stress is the result of a redox imbalance between the generation of reactive oxygen species (ROS) and the compensatory response from the endogenous anti-oxidant network. ROS are now known to act as secondary messengers during I/R (9), and it was reported that dietary flavonoids such as quercetin can block ROS generation (10). Furthermore, a study showed that quercetin can reduce the activation of apoptosis in focal cerebral ischemia in rat brain tissue and the mechanism may involve the brain-derived neurotrophic factor/tropomyosin receptor kinase B/phosphoinositide 3 kinase (PI3K)/Akt signaling pathway (11). Akt is a serine/threonine protein kinase, and the primary role of Akt signaling is to tansduct growth factor-mediated cell survival and block apoptosis, which is activated by phosphorylation. Pro-apoptotic factors, including Bcl-2-associated death 
promoter (Bad) and caspase- 9 and caspase-3 are modulated by Akt (12). The neuroprotective effect of Akt in cerebral ischemia has been extensively studied $(13,14)$. Temporary Akt phosphorylation of serine 473 was shown to occur after focal cerebral ischemia and phosphorylated Akt-positive cells exhibited decreased levels of DNA damage, indicating that the $\mathrm{PI} 3 \mathrm{~K} / \mathrm{Akt}$ pathway is involved in neuronal cell survival in I/R injury (15).

In view of these findings, the present study investigated whether oral administration of quercetin can reduce motor performance deficits and neuronal cell loss in the CA1 hippocampus using a rat model of global cerebral I/R injury.

\section{Materials and methods}

Animals. All experiments were performed on a total of 284 6-8 week-old male Sprague Dawley (SD) rats (280-320 g; Xi'an Experimental Animal Center of the Medical College, Xi'an Jiaotong University, Xi'an, China), housed at constant temperature $\left(21 \pm 1^{\circ} \mathrm{C}\right)$ and a 12 -h light/dark cycle (6 am to $6 \mathrm{pm})$. Food and water were available ad libitum. All animal handling as well as surgical and sacrification procedures were conducted in compliance with the guidelines of the Institutional Animal Care and Use Committee of Xi'an Jiaotong University (XJTU), which oversees conformity with national laws (Animal Welfare Act) and accreditation policies (Association for Assessment and Accreditation of Laboratory Animal Care). All experimental protocols in the present study were approved by the The Laboratory Animal Care and Use Committee of Xi'an Jiaotong University (Xi'an, China) Animals were transported to an approved animal unit 12-24 h prior to the experiment to reduce stress responses on the day of surgery.

Quercetin treatment. Quercetin ( $\geq 95 \%)$ was purchased from Sigma-Aldrich (St. Louis, MO, USA). Quercetin powder was dissolved in distilled water, and was stored at a final concentration of $1 \mathrm{~g} / \mathrm{ml}$. The solution was administered by oral gavage to the SD rats. Rats in the sham + vehicle group were given an equivalent volume of vehicle (distilled water, $10 \mathrm{ml} / \mathrm{kg}$, orally (p.o.); once daily). Volumes of drug solution required for the administration of 5,10 or $20 \mathrm{mg} / \mathrm{kg} /$ day quercetin equated to $3 \mathrm{ml}$ for a 300-g rat, which is an acceptable volume for oral gavage.

Rat model of global brain I/R. The global cerebral ischemia reperfusion model was established by a four-vessel occlusion (4-VO) ligation method as previously described (16). Rats were randomly assigned to four groups: Sham + vehicle group, $\mathrm{I} / \mathrm{R}+$ vehicle group, $\mathrm{I} / \mathrm{R}+5 \mathrm{mg} / \mathrm{kg} /$ day quercetin group and $\mathrm{I} / \mathrm{R}+10 \mathrm{mg} / \mathrm{kg} /$ day quercetin group. Prior to surgery, the animals were housed overnight with free access to tap water. Global cerebral ischemia was induced using the 4-VO method with slight modification (17). Briefly, on the first day, rats were anesthetized with chloral hydrate $(35 \mathrm{mg} / 100 \mathrm{~g}$ weight; Puzhen Bio Tech Ltd., Shanghai, China) and both vertebral arteries were electrocauterized permanently through the alar foramen of the first cervical vertebra. On the next day, under chloral hydrate anesthesia, bilateral carotid artery occlusion was performed by atraumatic micro-vessel clamps for $15 \mathrm{~min}$ to induce ischemia prior to the release of the carotid artery clamps and onset of the reperfusion. The neck incision was then closed with 3-0 aseptic non-absorbable silk sutures in a simple interrupted pattern. Sham-operated animals were treated similarly to those in the ischemic group, but neither the vertebral arteries nor the common carotid arteries were occluded. The rectal temperature was maintained at $36-37^{\circ} \mathrm{C}$ for all animals throughout the experiment. The successful establishment of the global cerebral I/R model was verified by identifying certain symptoms, including mydriasis, tachypnea, pale eyeballs and lip cyanosis.

Neurological score assessment. An independent observer serially evaluated the neurological functional outcome after graded injury using the Neuro Deficit Score (NDS) system as described previously (18). Neurologic evaluation was performed once daily at the same time (5:00 p.m.) by the same investigator, who was unaware of the group assignment at 12, 24 and $48 \mathrm{~h}$ after I/R injury. The NDS ranges from 80 (best) to 0 (brain dead) and it includes a sub-score of general behavioral deficits: Consciousness as normal, stuporous or unresponsive and arousal with eye opening and respiration as normal, abnormal (hypo- or hyperventilation) or absent. Brainstem function sub-scores are assessed with: i) Olfaction as response to smell of food; ii) vision, as head movement to light; presence of iii) pupillary light reflex; iv) corneal reflex; v) startle reflex; response to vi) whisker stimulation and vii) swallowing liquids or solids were also tested. Sub-score in motor assessment included strength testing as normal, abnormal (either stiff or weak) and absence of movement. All randomized animals were evaluated at baseline to ensure a normal neurologic score.

Morris water maze (MWM). The Morris water maze assay was executed as previously described $(19,20)$. The MWM consists of a black circular pool $(180 \mathrm{~cm}$ diameter, $45 \mathrm{~cm}$ high) filled with water (30 cm depth) at $22 \pm 1^{\circ} \mathrm{C}$ and is virtually divided into four equivalent quadrants: North-east (NE), north-west (NW), south-east (SE) and south-west (SW). A 2-cm submerged escape platform (12 cm diameter) was placed in the middle of one of the quadrants equidistant from the sidewall and the center of the pool. The pool was surrounded by several distal visual cues. Animal behavior was monitored by a video camera and quantified using Ethovision v1.90 (Noldus, Wageningen, The Netherlands). Prior to starting the MWM acquisition phase, animals received a cued learning training to instruct the animals on the procedures required to learn the MWM task. For cued learning, the pool is the same as that in the MWM version except that the platform is flagged above the water by $\sim 12 \mathrm{~cm}$ and curtains are closed around the maze to avoid access to distal visual cues. To ensure that rats are using the flagged cue to locate the platform, the location of the goal and the starting point were both moved to new positions during each trial. Four trials per day were performed until the rat reached the platform in two consecutive trials with a latency of $<20 \mathrm{sec}$. After completing cued learning, rats were trained following the standard MWM learning protocol consisting of four consecutive trials during three days, starting randomly from one of the four quadrants each time. A trial began by placing the rat into the water facing the wall of the pool. The rat was guided to the platform if it failed to escape 
within $90 \mathrm{sec}$ and was allowed to stay on the platform for $20 \mathrm{sec}$ maximum prior to returning to its cage for a new trial (inter-trial interval, $20 \mathrm{sec}$ ). On day four, a probe trial was performed, in which the escape platform was removed from the pool and the rat was allowed to swim for $60 \mathrm{sec}$. The trial began with the rat in the quadrant opposite the usual platform location. The time spent in each quadrant was recorded. In order to discriminate against the chance level, the percentage of time spent in the target quadrant over the total time spent in the target and the opposite quadrant (chance level at 50\%) was determined. The acquisition learning phase lasting 3 days provided a measure of spatial learning and reference memory, while the probe trial at day four measured spatial memory and retrieval capabilities.

Pathological analysis. Pathological Analysis was performed after $24 \mathrm{~h}$ of I/R injury. Hematoxylin and eosin (H\&E; Puzhen Bio Tech Ltd.) staining was performed according to standard protocols. Briefly, brain tissues were fixed in $4 \%(\mathrm{w} / \mathrm{v})$ paraformaldehyde and embedded in paraffin and then cut into $4-\mu \mathrm{m}$ sections. The slices underwent hematoxylin and eosin staining, respectively, for 20 and $2 \mathrm{~min}$ following de-waxing. After H\&E staining, images were captured using a microscope (Nikon Eclipse 80i; Nikon, Tokyo, Japan) to assess morphological differences among the experimental groups.

Apoptosis in brain tissues due to I/R injury was examined by terminal dUTP nick end labeling (TUNEL) analysis. Brain tissues were resected, fixed in paraformaldehyde for $24 \mathrm{~h}$, imbedded in paraffin and $5-\mu \mathrm{m}$ sections were prepared. The TUNEL assay was performed using an apoptag peroxidase in situ apoptosis detection kit (Chemicon International, Temecula, CA, USA). Briefly, the sections were digested using proteinase $\mathrm{K}$ and the endogenous peroxidase activity was blocked using 3\% hydrogen peroxide in phosphate-buffered saline (PBS). The sections were then placed in equilibration buffer and incubated with working strength of TdT enzyme in a humidifying chamber at $37^{\circ} \mathrm{C}$ for $1 \mathrm{~h}$. The reaction was terminated with a stop/wash buffer provided with the kit. The apoptotic nuclei were identified by detection of direct immuno peroxidase staining of digioxigenin-labeled DNA in the test sections.

Assessment of hippocampal cell viability and apoptosis. At $24 \mathrm{~h}$ following I/R injury, 24 rats (n=6/group) were intraperitoneally anesthetized and sacrificed by cervical dislocation to obtain the brain. The hippocampus was isolated and placed in a petri dish containing cold PBS $\left(0-4^{\circ} \mathrm{C}\right)$ on ice. The cerebral cortex $(0.5 \times 0.5 \mathrm{~cm})$ between the optic chiasma and mamillary body was isolated and stored on ice in a centrifuge tube containing PBS $\left(0-4^{\circ} \mathrm{C}\right)$. Single-cell suspensions were prepared within $30 \mathrm{~min}$ and the cell density was adjusted to $1 \times 10^{6} / \mathrm{ml}$. A total of $100 \mu \mathrm{l}$ cell suspension was placed in a $5-\mathrm{ml}$ test tube and mixed with $5 \mu \mathrm{l}$ Annexin V/fluorescein isothiocyanate and $10 \mu \mathrm{l}$ propidium iodide $(20 \mu \mathrm{l} / \mathrm{ml})$. The mixture was incubated in the dark at room temperature for $15 \mathrm{~min} .400 \mu \mathrm{l}$ PBS was added to the tube and cells were analyzed by flow cytometry (FACSCalibur; BD Biosciences, Franklin Lakes, NJ, USA).

Measurement of ROS. To assess ROS formation, 2',7'-dichlorofluorescein diacetate (DCFH-DA; Sigma-Aldrich) was used

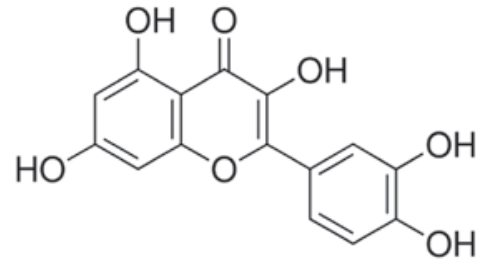

Figure 1. Chemical structure of quercetin.

as previously descried (21). Briefly, the hippocampus was isolated, homogenized using a manual glass homogenizer, and incubated with DCFH-DA $(100 \mu \mathrm{M})$ at $37^{\circ} \mathrm{C}$ for $30 \mathrm{~min}$. Cooling of the reaction mixture in ice terminated the reaction. The formation of the oxidized fluorescent derivative (DCF) was monitored at excitation and emission wavelengths of 488 and $525 \mathrm{~nm}$, respectively, using a fluorescence spectrophotometer (SP-Max 3500FL; Shanghai Flash Spectrum Bio Tech Co., Ltd., Shanghai, China). The free radical content was quantified using a DCF standard curve and results were expressed as nanomoles of DCF formed per milligram of protein.

Western blotanalysis. At $24 \mathrm{hfollowing} \mathrm{I} / \mathrm{R}$ injury, rats were intraperitoneally anesthetized and sacrificed by cervical dislocation to obtain the brain. Brain tissues (comprising the cortex and striatum) were collected and stored at $-80^{\circ} \mathrm{C}$. Tissues were harvested and resuspended in lysis buffer, washed with ice-cold PBS and lysed in extraction buffer, containing $40 \mathrm{mmol} / \mathrm{l}$ Tris- $\mathrm{HCl}$ (pH 7.5), $150 \mathrm{mmol} / 1 \mathrm{KCl}, 1 \mathrm{mmol} / \mathrm{l} \mathrm{EDTA}, 1 \%$ Triton X-100, $100 \mathrm{mmol} / 1 \mathrm{NaVO}_{3}, 1 \mathrm{mmol} / \mathrm{l} \mathrm{PMSF}$, supplemented with the protease inhibitor cocktail (Roche, Basel, Switzerland). The protein $(50 \mu \mathrm{g})$ was separated on 10\% SDS-PAGE gels and transferred onto nitrocellulose membranes. The membranes were blocked with 5\% non-fat milk in Tris-buffered saline (TBS) at $37^{\circ} \mathrm{C}$. Rabbit anti-Bcl-2 polyclonal antibody $(1: 1,000$; Cell Signaling Technology, Danvers, MA, USA; cat no. 2876), rabbit anti-Bcl-xL polyclonal antibody (1:1,000; Cell Signaling Technology, cat no. 2762), rabbit anti-Bad monoclonal antibody (1:1,000; Cell Signaling Technology, Danvers, MA, USA, cat no. 9293), rabbit phospho (p-)Bad (Ser136) monoclonal antibody (1:1,000; Cell Signaling Technology, Danvers, MA, USA, cat no. 4366), rabbit anti-survivin polyclonal antibody (1:1,000; Cell Signaling Technology, Danvers, MA, USA, cat no. 2803), rabbit anti-Akt polyclonal antibody (1:1,000; Cell Signaling Technology, Danvers, MA, USA, cat no. 9272), rabbit anti-p-Akt (Ser473) monoclonal antibody (1:1,000; Cell Signaling Technology, Danvers, MA, USA, cat no. 4060), and rabbit anti- $\beta$-actin monoclonal antibody $(1: 1,000$; Cell Signaling Technology, Danvers, MA, USA, cat no. 4967) were diluted in freshly prepared PBS containing 3\% skimmed milk powder, and blots were incubated in primary antibodies at $4^{\circ} \mathrm{C}$ overnight. Horseradish peroxidase-linked goat anti-rabbit $\operatorname{IgG}$ antibody (1:5,000; Beijing Biosynthesis Biotechnology Co., Ltd. Beijing, Cihna; cat. no. bs-0295G-HRP) was used as a secondary antibody in TBS containing 5\% non-fat milk and the blots were incubated for $3 \mathrm{~h}$ at room temperature. Antigen-antibody complexes were detected using an enhanced chemiluminescence kit (ECL Plus; GE Healthcare Life Sciences, Chalfont, $\mathrm{UK}$ ), and bands were analyzed using Image J version $1.42 \mathrm{q}$ software (National Institutes of Health, Bethesda, MD, USA). 


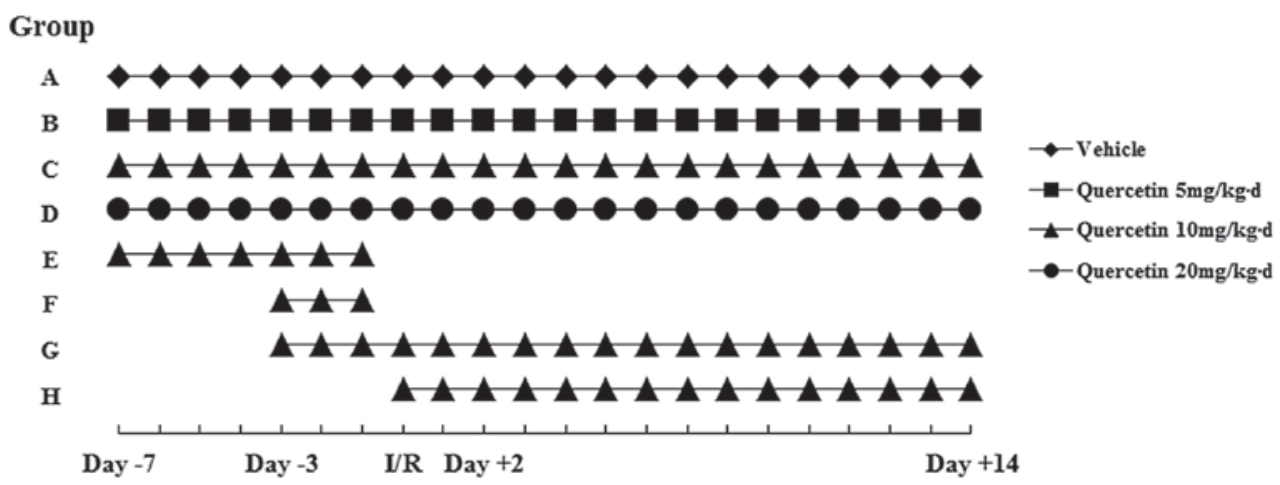

Figure 2. Animal groups for mortality assay. A total of 112 rats were randomly divided into eight groups: Rats treated with (A) vehicle; (B) $5 \mathrm{mg} / \mathrm{kg} / \mathrm{d}$ quercetin; (C) $10 \mathrm{mg} / \mathrm{kg} / \mathrm{d}$ quercetin; (D) $20 \mathrm{mg} / \mathrm{kg} / \mathrm{d}$ quercetin; (E) $10 \mathrm{mg} / \mathrm{kg} / \mathrm{d}$ quercetin from day -7 to I/R operation; (F) $10 \mathrm{mg} / \mathrm{kg} / \mathrm{d}$ quercetin from day -3 to I/R operation; (G) $10 \mathrm{mg} / \mathrm{kg} / \mathrm{d}$ quercetin from day -3 to +14 of $\mathrm{I} / \mathrm{R}$ operation; (H) $10 \mathrm{mg} / \mathrm{kg} / \mathrm{d}$ quercetin from $\mathrm{I} / \mathrm{R}$ operation to day+14. I/R, ischemia/reperfusion.
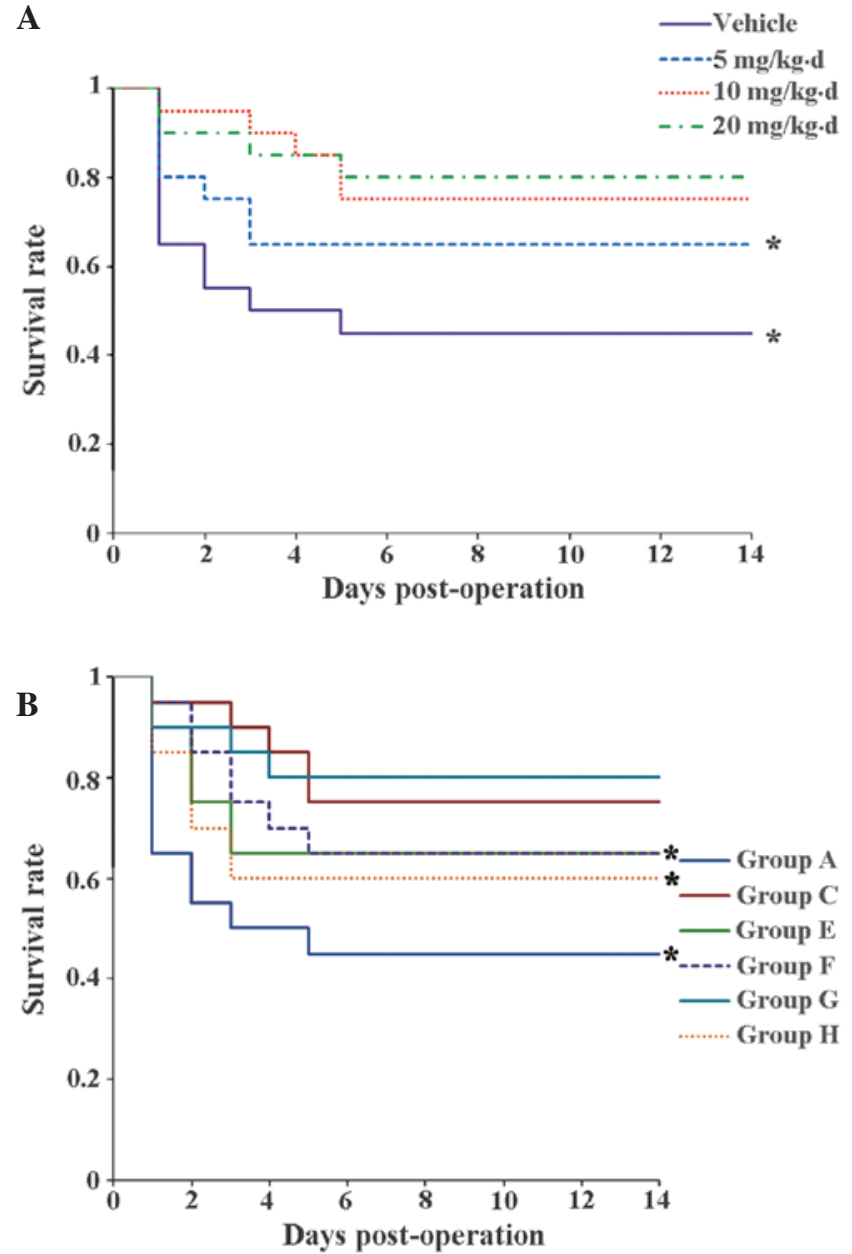

Figure 3. Survival rates of rats which underwent I/R with or without quercetin treatment. Quercetin treatment significantly decreased I/R-induced death. Rats treated with (A) vehicle; (B) $5 \mathrm{mg} / \mathrm{kg} / \mathrm{d}$ quercetin; (C) $10 \mathrm{mg} / \mathrm{kg} / \mathrm{d}$ quercetin; (D) $20 \mathrm{mg} / \mathrm{kg} / \mathrm{d}$ quercetin; (E) $10 \mathrm{mg} / \mathrm{kg} / \mathrm{d}$ quercetin from day -7 to $\mathrm{I} / \mathrm{R}$ operation; (F) $10 \mathrm{mg} / \mathrm{kg} / \mathrm{d}$ quercetin from day -3 to $\mathrm{I} / \mathrm{R}$ operation; (G) $10 \mathrm{mg} / \mathrm{kg} / \mathrm{d}$ quercetin from day -3 to +14 of I/R operation; $(\mathrm{H}) 10 \mathrm{mg} / \mathrm{kg} / \mathrm{d}$ quercetin from $\mathrm{I} / \mathrm{R}$ operation to day +14 . I/R, ischemia/reperfusion. $\mathrm{P}<0.05$, vs. Group A.

Statistical analyses. Unless otherwise indicated, values are expressed as the mean \pm standard error of the mean. Data were analyzed using SPSS 20.0 software for Windows (International Business Machines, Armonk, NY, USA). Differences between groups were analyzed using a one-way analysis of variance, and when significant, $t$ tests were employed for post hoc comparisons. $\mathrm{P}<0.05$ was considered to indicate a statistically significant difference between values.

\section{Results}

Quercetin treatment increases survival of rats following $I / R$. Prior to specific studies on the protective effect of quercetin on neuronal cells following $\mathrm{I} / \mathrm{R}$, an experiment was performed to confirm an appropriate concentration of quercetin for the treatment of cerebral I/R injury. The experimental design is illustrated in Fig. 2. Rats were divided into four groups: A, $\mathrm{I} / \mathrm{R}+$ vehicle; $\mathrm{B}, \mathrm{I} / \mathrm{R}+$ quercetin $5 \mathrm{mg} / \mathrm{kg} /$ day; $\mathrm{C}, \mathrm{I} / \mathrm{R}+$ quercetin $10 \mathrm{mg} / \mathrm{kg} /$ day; and $\mathrm{D}, \mathrm{I} / \mathrm{R}+$ quercetin $20 \mathrm{mg} / \mathrm{kg} /$ day. All rats were given individual treatment from 7 days prior to I/R to 14 days after I/R. As shown in Fig 3A, quercetin treatment significantly decreased I/R-induced death. Survival rates in groups B, C and D were significantly higher than that in group A; however, there was no significant difference between groups $\mathrm{C}$ and $\mathrm{D}$. In the sham group treated with quercetin (20 mg/kg/day for 21 days), none of the animals died (results not shown). The second experiment was executed to identify the appropriate timing of quercetin treatment to achieve a reduction of $\mathrm{I} / \mathrm{R}$ injury. All rats were treated with quercetin (10 $\mathrm{mg} / \mathrm{kg} /$ day) for different periods; rats were divided into four groups: $\mathrm{E}$, from day -7 until I/R; F, from day -3 until I/R; $\mathrm{G}$, from day -3 to day $+14 ; \mathrm{H}$, from $\mathrm{I} / \mathrm{R}$ to day +14 (Fig. 2 ). The survival rates in groups $\mathrm{C}$ and $\mathrm{G}$ were higher than those in all other groups with the same concentration of quercetin treatment. No significant difference was detected between group C and group $\mathrm{G}$, suggesting that treatment time may not influence the motility of rats after $\mathrm{I} / \mathrm{R}$. Therefore, rats were treated with 5 or $10 \mathrm{mg} / \mathrm{kg} /$ day quercetin from day -3 until the end of the experiments.

Motility is a critical indicator to evaluate neuroprotective effects following I/R injury (22). To assess the neuroprotective effect of quercetin, motility was assessed using two experiments in the present study.

Oral administration of quercetin attenuates $I / R$-induced performance deficits. Toevaluate the functional neuroprotective 

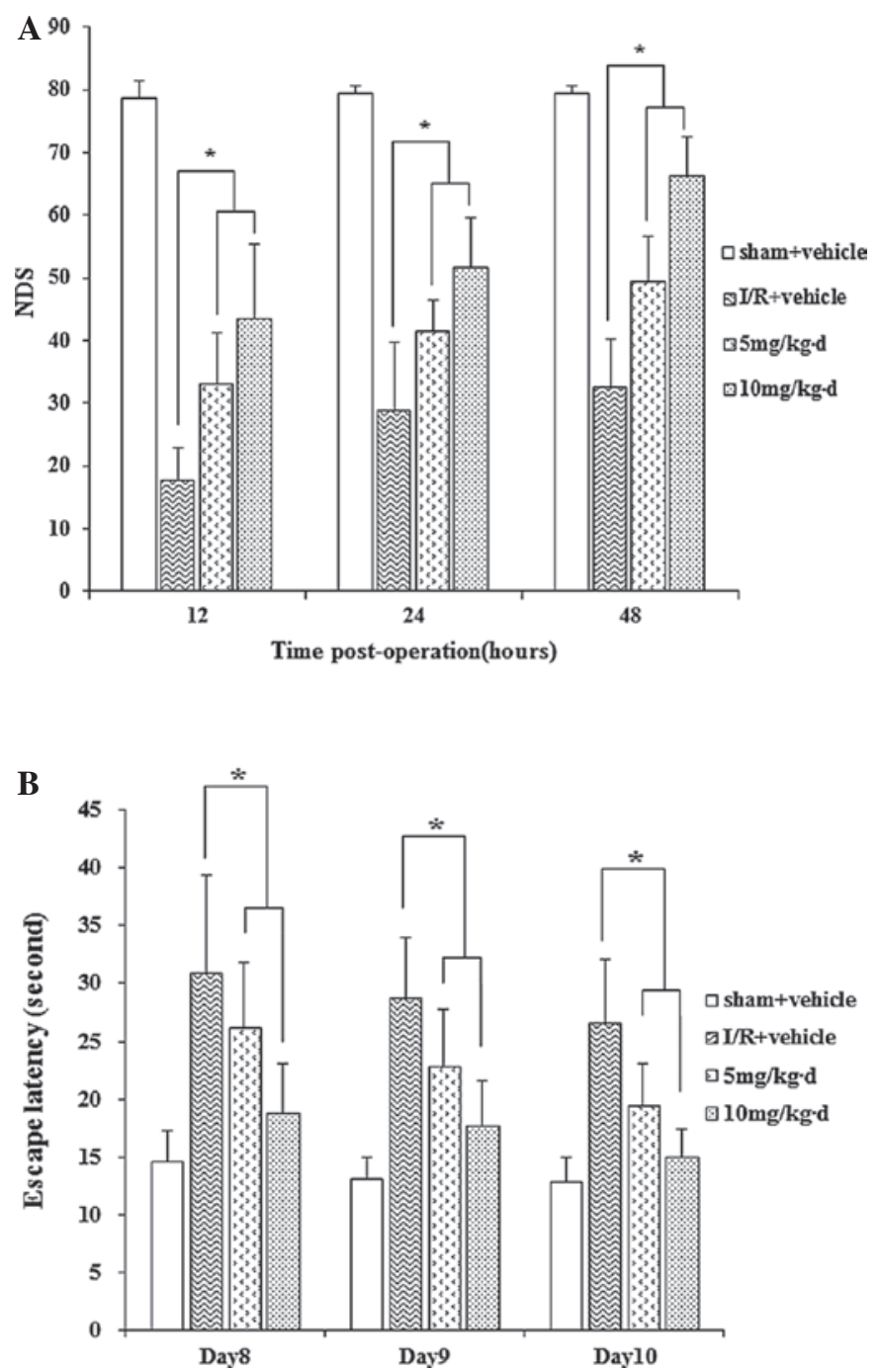

Figure 4. Oral administration of quercetin attenuates I/R-induced deficits in performance and spatial learning. (A) Oral administration of quercetin attenuated I/R-induced performance deficits. (B) Quercetin dose-dependently improved the response latency. Error bars indicate standard deviation ( ${ }^{*} \mathrm{P}<0.05$ vs. any other group). I/R, ischemia/reperfusion; NDS, neuro deficit score.

effect of quercetin in global cerebral I/R injury, neurological evaluation was performed at 12, 24 and $48 \mathrm{~h}$ after cerebral I/R injury.

NDS was significantly decreased at 12,24 and $48 \mathrm{~h}$ in I/R rats compared with those that underwent quercetin treatment and I/R (Fig. 4A). Compared to the sham + vehicle group, $15 \mathrm{~min}$ cerebral I/R caused a significant decrease in NDS (78.8 \pm 2.7 vs. $17.6 \pm 5.4$; $79.4 \pm 1.3$ vs. $29.0 \pm 10.8$; and $79.4 \pm 1.3$ vs. $32.6 \pm 7.8$ at 12,24 and $48 \mathrm{~h}$, respectively; $\mathrm{P}<0.05)$. Treatment with 5 and $10 \mathrm{mg} / \mathrm{kg} /$ day quercetin significantly increased the NDS caused by I/R $(33.2 \pm 8.1$ and $43.4 \pm 12.1$ vs. $17.6 \pm 5.4$; $41.6 \pm 4.9$ and $51.8 \pm 7.8$ vs. $29.0 \pm 10.8 ; 49.4 \pm 7.2$ and $66.2 \pm 6.2$ vs. $32.6 \pm 7.8$ at 12,24 and 48 h, respectively; $\mathrm{P}<0.05$ ).

Spatial learning and long-term memory is differently affected in $I / R+$ quercetin-treated and untreated $I / R$ rats. In the MWM test, differences between groups regarding cognitive abilities which are known to depend on the hippocampal function were assessed using the MWM task. In the cued learning task, animals in the sham + vehicle group reached the set goal

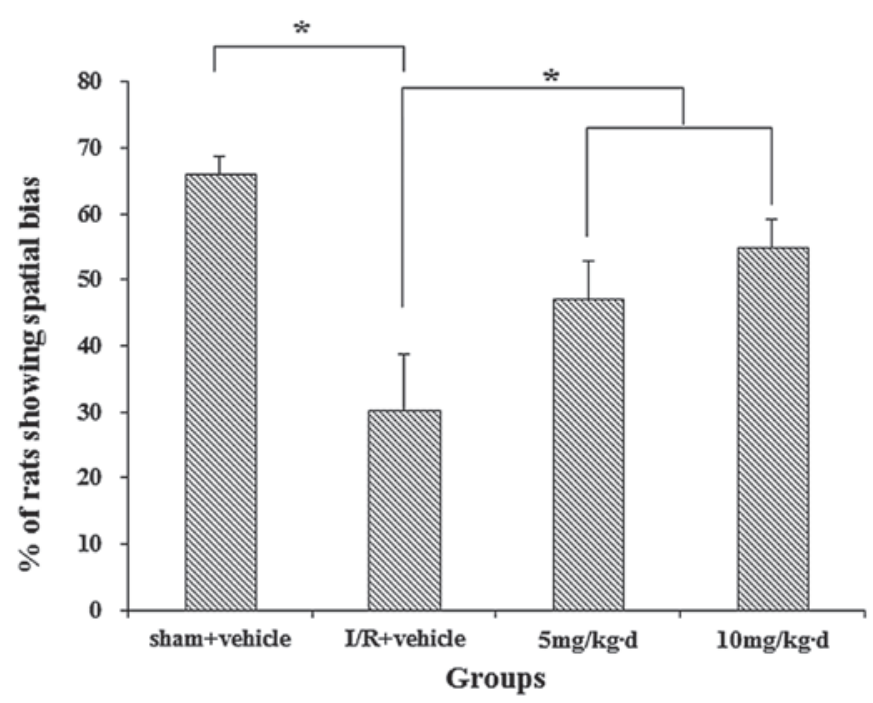

Figure 5. Quercetin treatment affects long-term spatial memory in I/R rats. Results of the Morris water maze test showing the percentage of rats that spent more time in the target quadrant than any other quadrant. Error bars indicate standard deviation ( $\mathrm{P}<0.05$ vs. any other group). I/R, ischemia/reperfusion.

on the first training day, while quercetin-treated and untreated I/R animals required 3 and 4 days of training, respectively. Seven days following cerebral I/R, animals performed the standard MWM acquisition task, which measures spatial learning. The escape latency was measured 8,9 and 10 days after ischemia (Fig. 4B). Animals in the sham + vehicle group performed better than I/R animals (14.5 $\pm 2.7,13.1 \pm 1.9$ and $12.8 \pm 2.1 \mathrm{sec}$ in the sham + vehicle group vs. $31.0 \pm 8.4$, $28.7 \pm 5.2$ and $26.6 \pm 5.5 \mathrm{sec}$ in the I/R group on day 8, 9 and 10, respectively). Significant differences were revealed in escape latency between the sham + vehicle and $I / R$ groups $(\mathrm{P}<0.05)$. This result confirmed that $\mathrm{I} / \mathrm{R}$ elicited significant deficits in spatial learning. At days 8, 9 and 10, the escape latency was differently affected in $\mathrm{I} / \mathrm{R}+$ quercetin-treated and I/R animals $(26.2 \pm 5.7,22.8 \pm 5.0$ and $19.5 \pm 3.6 \mathrm{sec}$ in the $5 \mathrm{mg} / \mathrm{kg} /$ day group and $18.8 \pm 4.3,17.8 \pm 3.9$ and $15.1 \pm 2.4 \mathrm{sec}$ in the $10 \mathrm{mg} / \mathrm{kg} /$ day group). Quercetin dose-dependently decreased the escape latency, and the two doses (5 and $10 \mathrm{mg} / \mathrm{kg} / \mathrm{day}$ ) caused a statistically significant effect in the escape latency at days 8 , 9 and 10 after $I / R(P<0.01$ vs. ischemic animals). Significant group differences were revealed during the acquisition phase trial between $\mathrm{I} / \mathrm{R}+$ quercetin-treated groups (5 and $10 \mathrm{mg} / \mathrm{kg} /$ day) and the I/R group. These results revealed that Quercetin-treated rats showed an attenuated spatial learning deficit.

In the probe MWM trial, similar patterns were observed in terms of the percentages of animals spending more time in the target quadrant than in any other quadrant (Fig. 5), referred to as spatial bias. Significant differences in spatial bias were identified between the sham + vehicle group and rats subjected to $\mathrm{I} / \mathrm{R}(\mathrm{P}<0.05)$. This may be due to an $\mathrm{I} / \mathrm{R}$-induced impairment in spatial memory retention. By contrast, quercetin-treated animals more effectively discriminated the target quadrant, and the fraction of rats showing spatial bias after quercetin treatment was significantly higher than that in the vehicle-treated I/R group. Compared with untreated animals, 


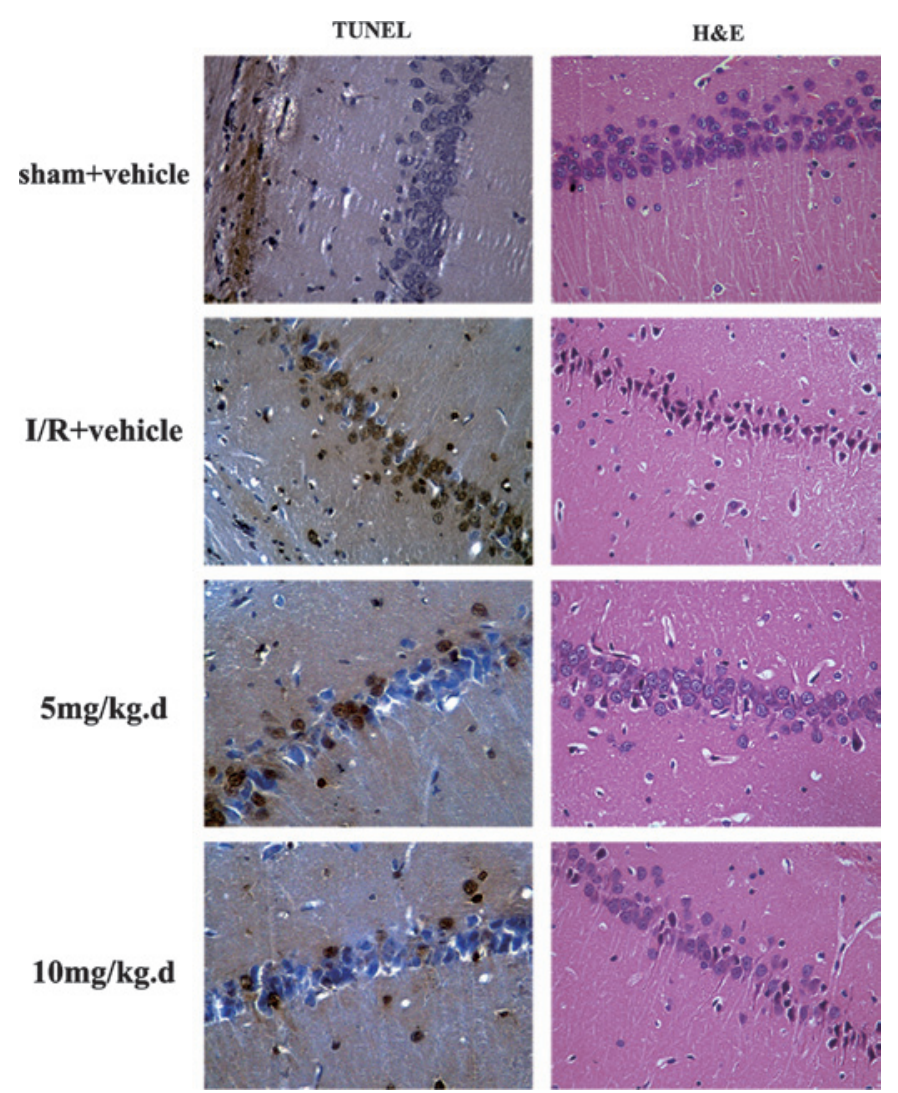

Figure 6. Dose-dependent reduction of I/R-induced hippocampal neuron loss by oral administration of quercetin. The TUNEL-positive cells were significantly decreased in the quercetin-treated group. Such neuroprotective effect was also illustrated using H\&E staining (magnification, $\mathrm{x} 400$ ). H\&E, hematoxylin and eosin; TUNEL, terminal deoxynucleotidyl transferase dUTP nick end labeling; I/R, ischemia/reperfusion.

quercetin-treated animals subjected to I/R spent more time in the target quadrant than in any other quadrant $(\mathrm{P}<0.05)$. The fact that the performance of quercetin-treated rats improved in the probe trials may support that quercetin improves long-term spatial memory retention.

Quercetin reduces neuronal cell apoptosis caused by I/R injury. To detect the effect of quercetin on histological alteration after cerebral I/R injury in the hippocampus, dose-response associations regarding the neuroprotective effects of pre-treatment with quercetin on the CA1 hippocampus of rats subjected to I/R were examined. Four groups of rats were orally administered once daily with vehicle or quercetin (5 or $10 \mathrm{mg} / \mathrm{kg} /$ day). All animals were subjected to $15 \mathrm{~min}$ ischemia $24 \mathrm{~h}$ after the last administration of water or quercetin. Neuronal cell loss in the hippocampus was assessed $24 \mathrm{~h}$ after I/R by counting the number of apoptotic cells in brain sections from these structures using the TUNEL assay (Fig. 6) and FACS (Fig. 7). Quercetin treatment caused a dose-dependent increase in neuronal cell survival in the dorsal hippocampus. As shown in Fig. 6, the TUNEL-positive cells were significantly decreased in the quercetin-treated group. This neuroprotective effect was also illustrated by H\&E staining (Fig. 6). According to the FACS assay, the ratios of apoptotic cells were $3.57 \pm 0.38 \%$ for the sham + vehicle treatment group, $35.56 \pm 5.39 \%$ for the $\mathrm{I} / \mathrm{R}+$ vehicle treatment group, $19.28 \pm 3.64 \%$ for the $\mathrm{I} / \mathrm{R}+5 \mathrm{mg} / \mathrm{kg} /$ day quercetin treatment
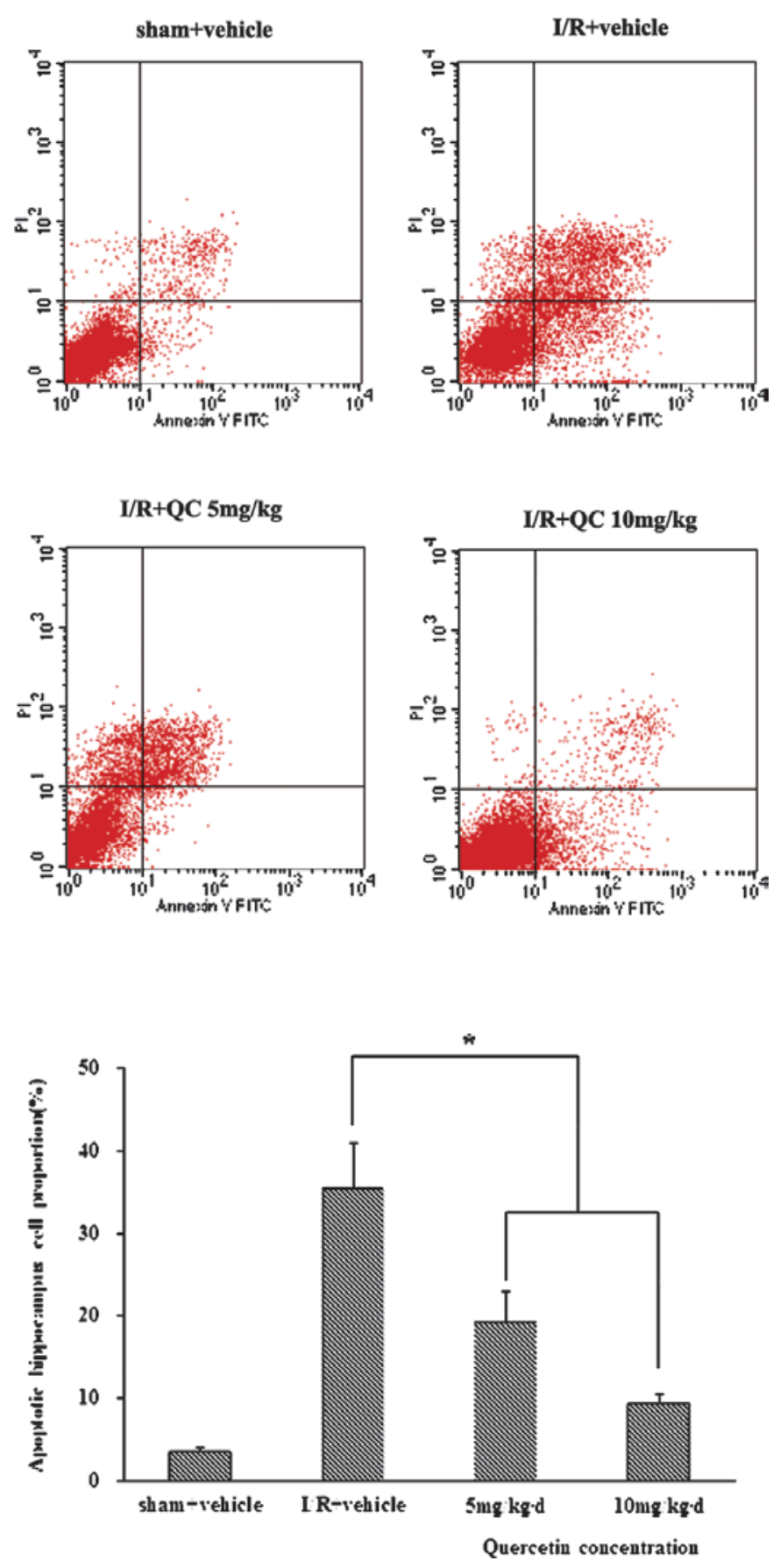

Figure 7. Quercetin reduces neuronal cell apoptosis caused by I/R injury. $24 \mathrm{~h}$ after $\mathrm{I} / \mathrm{R}$, the apoptotic rate was $3.57 \pm 0.38 \%$ for the sham + vehicle treatment group, $35.56 \pm 5.39 \%$ for the I/R + vehicle treatment group, $19.28 \pm 3.64 \%$ for the $\mathrm{I} / \mathrm{R}+5 \mathrm{mg} / \mathrm{kg} / \mathrm{d}$ quercetin treatment group and $9.41 \pm 1.16 \%$ for the $\mathrm{I} / \mathrm{R}+10 \mathrm{mg} / \mathrm{kg} / \mathrm{d}$ quercetin treatment group. Error bars indicate standard deviation $(\mathrm{P}<0.05$ vs. I/R group). I/R, ischemia/reperfusion; PI, propidium iodide; FITC, fluorescein isothiocyanate; QC, quercetin.

group and $9.41 \pm 1.16 \%$ for the $\mathrm{I} / \mathrm{R}+10 \mathrm{mg} / \mathrm{kg} /$ day quercetin treatment group (Fig. 7). Increasing the dose of quercetin to $40 \mathrm{mg} / \mathrm{kg} /$ day did not produce any further improvement of neuronal cell survival (data not shown).

Quercetin-treatment decreases ROS formation. The fluorogenic compound DCFH-DA is one of the most prominent markers to reflect the overall oxidative status in cells. Within the cell, esterases cleave the acetate groups on DCFH-DA, thus 


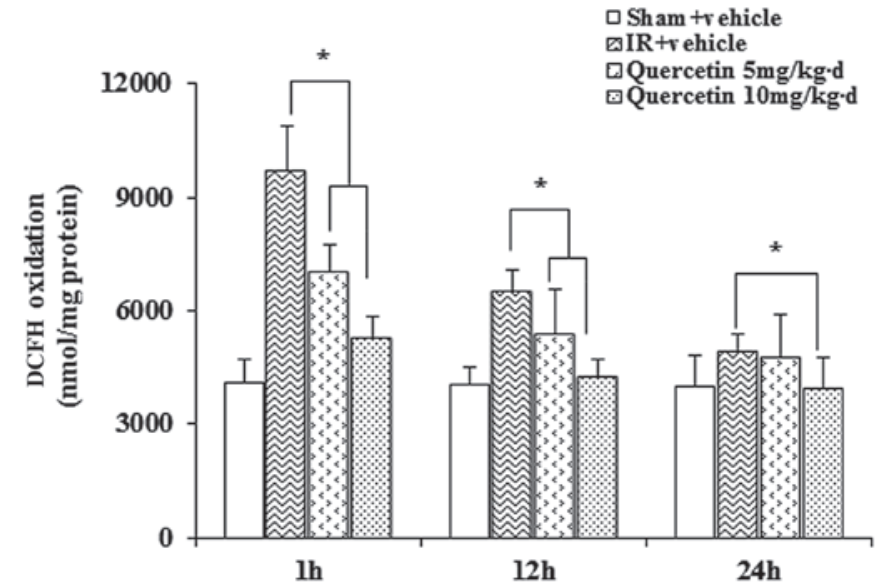

Figure 8. Effect of quercetin on ROS generation in hippocampi of rats after global cerebral ischemia. ROS levels were assessed using a DCFH assay. The I/R-induced ROS over-production at $1 \mathrm{~h}$ of reperfusion was significantly decreased by quercetin in the hippocampus. Values are expressed as the mean \pm standard deviation $(\mathrm{n}=6$ each). Error bars indicate standard deviation ("P<0.05). ROS, reactive oxygen species; I/R, ischemia/reperfusion; DCFH, dichlorodihydrofluorescein.

trapping the reduced probe (DCFH) intracellularly. ROS in the cells lead to the oxidation of DCFH, yielding the fluorescent product DCF. As shown in Fig. 8, rats that had undergone transient global cerebral ischemia for 15 min followed by $1 \mathrm{~h}$ of reperfusion exhibited a significantly increase in ROS production in the hippocampus $(\mathrm{P}<0.01)$ as compared with levels in vehicle-treated sham animals. By contrast, the I/R induced ROS overproduction following 12 and $24 \mathrm{~h}$ of reperfusion was significantly decreased by quercetin in the hippocampus $(\mathrm{P}<0.01$; Fig. 8). No alterations in DCFH levels were observed in sham animals.

Quercetin modulates the expression of anti-apoptotic proteins following $I / R$ in a fashion that is characteristic for neuroprotection. To reveal the effect of quercetin on apoptosis-associated proteins in brain tissues after I/R injury, the expression of Bcl-2, Bcl-xL, survivin and cleaved caspase-3 was assessed in the hippocampus $24 \mathrm{~h}$ following I/R (Fig. 9). I/R decreased the expression levels of $\mathrm{Bcl}-2$ and $\mathrm{Bcl}-\mathrm{xL}$ as compared with those in the sham + vehicle group, while it increased the levels of cleaved caspase-3. Administration of quercetin prior to $\mathrm{I} / \mathrm{R}$ (quercetin-I/R) was able to significantly increase the expression of Bcl-2, Bcl-xL and survivin, as well as restore levels of cleaved caspase-3 (Fig. 9).

Effects of quercetin on Akt signaling in brain after cerebral I/R injury. In the final experiment, the effects of quercetin on Akt signaling as well as its mainly apoptosis-associated substrate, Bad, were assessed. As shown in Fig. 10, at $24 \mathrm{~h}$ after I/R, the phosphorylation of Akt as well as Bad levels were significantly upregulated in the hippocampi of animals in the quercetin-treated $\mathrm{I} / \mathrm{R}$ group compared with those in the vehicle-treated I/R group.

\section{Discussion}

The neuroprotective effects of quercetin on the central nervous system have been previously reported, and most of these studies

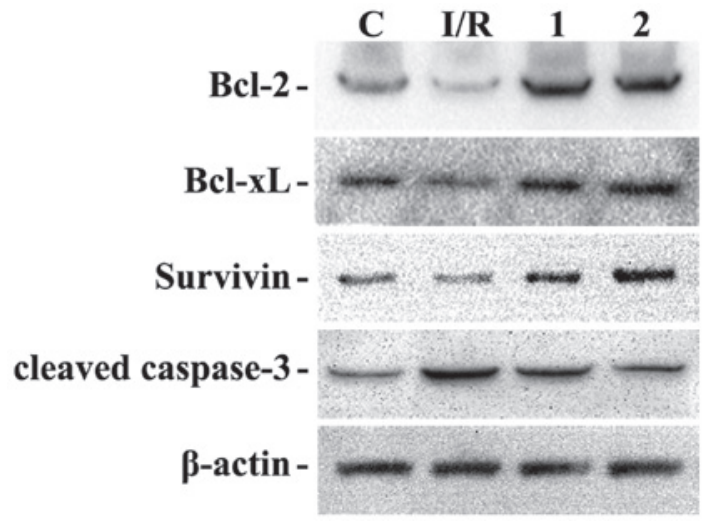

Figure 9. QC modulates the expression of anti-apoptotic genes following I/R in a fashion characteristic for neuroprotection. $\mathrm{C}$, sham + vehicle group; $\mathrm{I} / \mathrm{R}$, I $/ \mathrm{R}$ group; $1, \mathrm{IR}+5 \mathrm{mg} / \mathrm{kg} / \mathrm{d}$ QC treatment; $2, \mathrm{IR}+10 \mathrm{mg} / \mathrm{kg} / \mathrm{d}$ QC treatment. $24 \mathrm{~h}$ after I/R, QC treatment significantly upregulated Bcl-2, Bcl-xL and survivin, while it downregulated cleaved caspase-3, which was enhanced by $\mathrm{I} / \mathrm{R}$ in the hippocampus compared with the sham + vehicle group. I/R, ischemia/reperfusion; QC, quercetin; Bcl-2, B-cell lymphoma 2; xL, extra large.
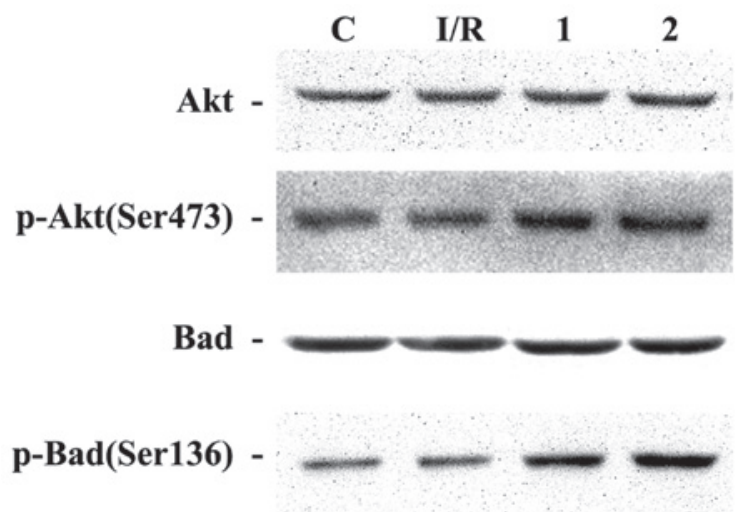

Figure 10. Effects of QC on Akt signaling and Akt-associated apoptosis modulators in the brain after cerebral I/R injury. $C$, sham + vehicle group; I/R, I/R group; $1, \mathrm{IR}+5 \mathrm{mg} / \mathrm{kg} / \mathrm{d}$ QC treatment; $2, \mathrm{IR}+10 \mathrm{mg} / \mathrm{kg} / \mathrm{d}$ QC treatment. $24 \mathrm{~h}$ after I/R, QC treatment upregulated phosphorylation of Akt as well as Bad in the hippocampus compared with vehicle treatment. I/R, ischemia/reperfusion; QC, quercetin; Bad, B-cell lymphoma 2-associated death promoter.

were based on focal cerebral ischemia models $(11,23,24)$. The advantage of the focal ischemia model is that the injury can be evaluated, which cannot be performed in the global brain ischemia model. However, global brain ischemia occurs in a certain percentage of patients with heart dysfunction, i.e. myocardial infarction, who usually contract reperfusion injury-induced brain damage. Therefore, global brain ischemia is another prevalent brain $\mathrm{I} / \mathrm{R}$ injury in the clinic. Previous studies have demonstrated that cerebral I/R injury causes neurological deficits and neuronal cell apoptosis has an important role in the evolution of $I / R$ injury in the brain $(25,26)$. In the present study, treatment with quercetin was shown to significantly improve neurological function at 12,24 and $48 \mathrm{~h}$ after cerebral ischemia according to NDS. These results were in accordance with those of previous studies $(11,23)$; however, to the best of our knowledge, the present study was the first to report that quercetin protects certain neuronal cells from global cerebral I/R injury. 
Behavioral parameters are useful measures of functional deficits following experimental global cerebral ischemia and the degree of sensorimotor dysfunction as an important indicator of the severity of injury. The motor function was found to be disturbed by neurological evaluation. The present study proved that the motor function was impaired after ischemic insults in rats and was significantly ameliorated by treatment with quercetin. These findings are in accordance with earlier studies where motor deficits were attenuated by treatment with anti-oxidants $(4,11,23)$.

Hippocampus-dependent cognitive impairments were quantified using the MWM. It was found that compared with vehicle-treated animals, quercetin-treated rats more effectively learned and remembered tasks in the MWM. By contrast, I/R animals performed poorly at the acquisition phase of the task and failed memory retention tests. In the acquisition phase of the MWM task, quercetin-treated animals performed better than their I/R peers, which spent more time to locate the hidden platform, while only animals in sham + vehicle group showed successful preference for the target quadrant in the probe trial and animals that received $\mathrm{I} / \mathrm{R}$ only were impaired the most among all groups. Analysis of escape latency (quantified as the time required to locate the hidden platform) and spatial bias (defined as the percentage of time spent in the target area with regard to the time spent in all quadrants), which represent spatial learning and memory retention, respectively, clearly showed that animals subjected to I/R injury alone were the most seriously affected, while quercetin-treated animals performed better regarding preference for platform location and the target quadrant. The results showed that quercetin had a significant positive effect in terms of spatial learning and long-term memory retention. Therefore, the present study confirmed that quercetin treatment effectively reversed cognitive impairment following I/R injury. The findings suggested that further studies on quercetin are warranted to determine its usefulness as a potential agent for the prevention of $I / R$ injuries.

The behavioral impact is closely linked to the degree of neuronal dysfunction. Functional deficits are a common neurological sequel in animal models of cerebral ischemia. The present study demonstrated that treatment with quercetin has a far-reaching protective effect against the consequences of ischemic insults. A rat model of global cerebral I/R injury was used for the behavioral and biochemical tests, which is widely used to study the neuroprotective effect of quercetin, because it represents the biochemical and pathological features of stroke in humans, including oxidative stress, mitochondrial dysfunction and apoptosis (27). In response to the oxidative load in mitochondria, the outer membrane of mitochondria becomes permeabilized (7), resulting in the translocation of Bax from the cytosol to the mitochondria and the release of cytochrome $c$. Since delayed neuronal degeneration is mostly due to apoptosis, the present study further investigated the number of apoptotic cells by TUNEL staining. The results showed less TUNEL-positive cells in the quercetin treatment group compared with that in the vehicle-treated I/R rats. Previous studies reported decreased protein levels of Bcl-2 and increased protein levels of Bax and cleaved-caspase-3 in MCAO rats following $I / R(28,29)$. The present study demonstrated that quercetin treatment was associated with pronounced upregulation of $\mathrm{Bcl}-2, \mathrm{Bcl}-\mathrm{xL}$ and survivin, as well as downregulation of cleaved-caspase- 3 . These results provided further evidence that quercetin is able to reduce apoptosis following ischemia through inhibiting the mitochondria-dependent caspase-mediated apoptotic pathway.

ROS generation in endothelial cells during hypoxia/reoxygenation is well documented $(30,31)$. Excess ROS from endogenous sources can account for autocrine and paracrine cellular damage during reperfusion $(32,33)$. The results of the present study showed that ROS production in the brain increased after $1 \mathrm{~h}$ of reperfusion. Decreased ROS generation following treatment with quercetin indicated that the neuroprotection conferred by quercetin was due to its anti-oxidant effect of attenuating oxidative stress.

Accumulating evidence suggested that the PI3K/Akt signaling pathway has a crucial role in neuronal survival after cerebral ischemia. Previous studies have reported that activation of Akt signaling has neuroprotective effects against ischemic neuronal injury $(34,35)$. Several potential substrates for Akt that are associated with cell survival after cerebral I/R injury. To determine whether Akt activation is a major contributor to the anti-apoptotic effect of quercetin following ischemia, the phosphorylation of Akt after global cerebral I/R injury was assessed using western blot analysis. Treatment with quercetin significantly increased the expression of p-Akt compared with that in the vehicle-treated group. The expression of p-Akt downstream signaling proteins was further examined. Quercetin was shown to upregulate the phosphorylation level of Bad, suggesting that Bad is one of the downstream targets of quercetin-induced inhibition of Akt. Previous studies have shown that blocking PI3K/Akt signaling with the pharmacological inhibitor LY294002 significantly attenuated the anti-apoptotic effect of quercetin (11). The results of the present study suggested that the protective effect of quercetin against apoptosis induced by ischemia is mediated by the PI3K/Akt pathway.

Quercetin alters the expression of anti-apoptotic genes in response to I/R. The neuroprotective effects of flavonoids have been linked to the activation of pro-survival signaling mediated by the PI3/Akt and ERK pathways, which stimulate the expression of the prototypical anti-apoptotic genes $\mathrm{Bcl}-2$ and $\mathrm{X}$-linked inhibitor of apoptosis protein. I/R enhanced the expression of Bcl-2 mRNA in the dorsal hippocampus, which was completely reversed by quercetin (36). In situ hybridization histochemical studies have localized the increases in Bcl-2 mRNA following transient global cerebral ischemia to pyramidal neurons in the hippocampus, which are exquisitely sensitive to ischemic injury (37). Reversal of I/R-induced increases in $\mathrm{Bcl}-2$ gene expression by quercetin may therefore reflect a reduction in $N$-methyl-D-aspartate receptor-mediated cyclic adenosine monophosphate responsive element binding protein signaling (38).

In conclusion, the present study demonstrated that the oral administration of quercetin ( 5 and $10 \mathrm{mg} / \mathrm{kg} /$ day) prevented motor performance deficits and markedly attenuated neuronal cell loss in the dorsal hippocampus in a rat model of global cerebral I/R injury. The neuroprotection of quercetin is associated with the activation of PI3K/Akt signaling in response to ischemia-induced apoptosis. Further investigation into the underlying mechanisms of the anti-oxidant action of quercetin 
is required to determine whether it can be an effective drug for the prevention of brain injury following stroke.

\section{Acknowledgements}

This work was supported by grants from the Key Science and Technology Program of Shaanxi Province [no. 2010k15-03 (1)].

\section{References}

1. Cherubini A, Ruggiero C, Morand C, et al: Dietary antioxidants as potential pharmacological agents for ischemic stroke. Curr Med Chem 15: 1236-1248, 2008.

2. Harwood M, Danielewska-Nikiel B, Borzelleca JF, Flamm GW Williams GM and Lines TC: A critical review of the data related to the safety of quercetin and lack of evidence of in vivo toxicity, including lack of genotoxic/carcinogenic properties. Food Chem Toxicol 45: 2179-2205, 2007.

3. Lee JC, Kim J, Park JK, Chung GH and Jang YS: The antioxidant, rather than prooxidant, activities of quercetin on normal cells: Quercetin protects mouse thymocytes from glucose oxidase-mediated apoptosis. Exp Cell Res 291: 386-397, 2003.

4. Lapi D, Vagnani S, Pignataro G, esposito E, Paterni M and Colantuoni A: Protective effects of Quercetin on Rat Pial microvascular changes during transient bilateral common carotid artery occlusion and reperfusion. Front Physiol 3: 32, 2012.

5. Ghosh A, Sarkar S, Mandal AK and Das N: Neuroprotective role of nanoencapsulated quercetin in combating ischemia-reperfusion induced neuronal damage in young and aged rats. PLoS One 8: e57735, 2013.

6. Mattson MP, Duan W, Pedersen WA and Culmsee C: Neurodegenerative disorders and ischemic brain diseases. Apoptosis 6: 69-81, 2001.

7. Kowaltowski AJ, Castilho RF and Vercesi AE: Mitochondrial permeability transition and oxidative stress. FEBS Lett 495: 12-15, 2001.

8. Kuwana T, Mackey MR, Perkins G, et al: Bid, Bax and lipids cooperate to form supramolecular openings in the outer mitochondrial membrane. Cell 111: 331-342, 2002

9. Lei C, Deng J, Wang B, et al: Reactive oxygen species scavenger inhibits STAT3 activation after transient focal cerebral ischemia-reperfusion injury in rats. Anesth Analg 113: 153-159, 2011

10. Priyadarsini RV and Nagini S: Quercetin suppresses cytochrome P450 mediated ROS generation and NF kappaB activation to inhibit the development of 7,12-dimethylbenz a anthracene (DMBA) induced hamster buccal pouch carcinomas. Free Radic Res 46: 41-49, 2012.

11. Yao RQ, Qi DS, Yu HL, Liu J, Yang LH and Wu XX: Quercetin attenuates cell apoptosis in focal cerebral ischemia rat brain via activation of BDNF-TrkB-PI3K/Akt signaling pathway. Neurochem Res 37: 2777-2786, 2012.

12. Mayo LD and Donner DB: A phosphatidylinositol 3-kinase/Akt pathway promotes translocation of Mdm2 from the cytoplasm to the nucleus. Proc Natl Acad Sci USA 98: 11598-11603, 2001.

13. Janelidze S, Hu BR, Siesjo P and Siesjo BK: Alterations of Akt1 (PKBalpha) and p70 (S6K) in transient focal ischemia. Neurobiol Dis 8: $147-154,2001$

14. Shibata M, Yamawaki T, Sasaki T, et al: Upregulation of Akt phosphorylation at the early stage of middle cerebral artery occlusion in mice. Brain Res 942: 1-10, 2002.

15. Noshita N, Lewen A, Sugawara T and Chan PH: Evidence of phosphorylation of Akt and neuronal survival after transient focal cerebral ischemia in mice. J Cereb Blood Flow Metab 21: 1442-1450, 2001

16. Xue RL, He JX, Wang N, Yao FZ, Lv JR and Wu G: Relationship between transmembrane signal transduction pathway and DNA repair and the mechanism after global cerebral ischemia-reperfusion in rats. Neurosci Bull 25: 115-121, 2009.

17. Hadley G, Papadakis M and Buchan AM: A method of inducing global cerebral ischemia. Methods Mol Biol 1135: 111-120, 2014.

18. Geocadin RG, Ghodadra R, Kimura T, et al: A novel quantitative EEG injury measure of global cerebral ischemia. Clin Neurophysiol 111: 1779-1787, 2000.

19. Vorhees CV and Williams MT: Morris water maze: procedures for assessing spatial and related forms of learning and memory. Nat Protoc 1: 848-858, 2006.
20. Stackman RW Jr,Lora JC and Williams SB: Directional responding of C57BL/6J mice in the Morris water maze is influenced by visual and vestibular cues and is dependent on the anterior thalamic nuclei. J Neurosci 32: 10211-10225, 2012.

21. Simao F, Matte A, Matte C, et al: Resveratrol prevents oxidative stress and inhibition of $\mathrm{Na}(+) \mathrm{K}(+)$-ATPase activity induced by transient global cerebral ischemia in rats. J Nutr Biochem 22: 921-928, 2011.

22. Johansson SE, Larsen SS, Povlsen GK and Edvinsson L: Early MEK1/2 inhibition after global cerebral ischemia in rats reduces brain damage and improves outcome by preventing delayed vasoconstrictor receptor upregulation. PLoS One 9: e92417, 2014.

23. Ahmad A, Khan MM, Hoda MN, et al: Quercetin protects against oxidative stress associated damages in a rat model of transient focal cerebral ischemia and reperfusion. Neurochem Res 36: 1360-1371, 2011.

24. Pandey AK, Hazari PP, Patnaik R and Mishra AK: The role of ASIC1a in neuroprotection elicited by quercetin in focal cerebral ischemia. Brain Res 1383: 289-299, 2011

25. Rizk NN, Rafols JA and Dunbar JC: Cerebral ischemia-induced apoptosis and necrosis in normal and diabetic rats: effects of insulin and C-peptide. Brain Res 1096: 204-212, 2006.

26. Solaroglu I, Tsubokawa T, Cahill J and Zhang JH: Anti-apoptotic effect of granulocyte-colony stimulating factor after focal cerebral ischemia in the rat. Neuroscience 143 965-974, 2006

27. Yousuf S, Atif F, Ahmad M, et al: Resveratrol exerts its neuroprotective effect by modulating mitochondrial dysfunctions and associated cell death during cerebral ischemia. Brain Res 1250: 242-253, 2009

28. Wang R, Li G, Wang W and Li H: Effect of compound preparation Tongqiao Jiannao capsules on neural cell apoptosis and $\mathrm{Bcl}-2$ and Bax protein levels in a rat model of brain ischemia/reperfusion injury. Neural Regener Res 3: 871-874, 2008.

29. Babu PP, Yoshida Y, Su M, Segura M, Kawamura S and Yasui N: Immunohistochemical expression of Bcl-2, Bax and cytochrome c following focal cerebral ischemia and effect of hypothermia in rat. Neurosci Lett 291: 196-200, 2000.

30. Wang T, Gu J, Wu PF, et al: Protection by tetrahydroxystilbene glucoside against cerebral ischemia: involvement of JNK, SIRT1 and NF-kappaB pathways and inhibition of intracellular ROS/RNS generation. Free Radic Biol Med 47: 229-240, 2009.

31. Wu HW, Li HF, Wu XY, Zhao J and Guo J: Reactive oxygen species mediate ERK activation through different Raf-1-dependent signaling pathways following cerebral ischemia. Neurosci Lett 432: 83-87, 2008.

32. Wang Q, Sun AY, Simonyi A, et al: Ethanol preconditioning protects against ischemia/reperfusion-induced brain damage: Role of NADPH oxidase-derived ROS. Free Radic Biol Med 43: 1048-1060, 2007.

33. Liang JM, Xu HY, Zhang XJ, Li X, Zhang HB and Ge PF: Role of mitochondrial function in the protective effects of ischaemic postconditioning on ischaemia/reperfusion cerebral damage. J Int Med Res 41: 618-627, 2013.

34. Zhang L, Zhang ZG, Liu XS, Hozeska-Solgot A and Chopp M: The PI3K/Akt pathway mediates the neuroprotective effect of atorvastatin in extending thrombolytic therapy after embolic stroke in the rat. Arterioscler Thromb Vasc Biol 27: 2470-2475, 2007.

35. Endo H, Nito C, Kamada H, Nishi T and Chan PH: Activation of the Akt/GSK3beta signaling pathway mediates survival of vulnerable hippocampal neurons after transient global cerebral ischemia in rats. J Cereb Blood Flow Metab 26: 1479-1489, 2006.

36. Fang $X X$, Jiang XL, Han XH, Peng YP and Qiu YH: Neuroprotection of interleukin-6 against NMDA-induced neurotoxicity is mediated by JAK/STAT3, MAPK/ERK and PI3K/AKT signaling pathways. Cell Mol Neurobiol 33: 241-251, 2013.

37. Gu R, Liu M, Wang Y, Zhou Y and He H: Angiopoietin-1 mRNA and Bcl-2 expression following estradiol treatment in ovariectomized rats with focal cerebral ischemia/reperfusion injury. Neural Regener Res 4: 780-785, 2009.

38. Kitagawa K: CREB and cAMP response element-mediated gene expression in the ischemic brain. FEBS J 274: 3210-3217, 2007. 